Mathematical Modelling and Analysis

Volume 13 Number 1, 2008, Pages 7-16

(C) 2008 Technika ISSN 1392-6292 print, ISSN 1648-3510 online

\title{
INVERTIBILITY PROPERTIES OF MATRIX WIENER-HOPF PLUS HANKEL INTEGRAL OPERATORS
}

\author{
G. BOGVERADZE and L. P. CASTRO \\ Research Unit Matemática e Aplicações, Department of Mathematics, \\ University of Aveiro \\ 3810-193 Aveiro, Portugal \\ E-mail: giorgi@ua.pt; castro@ua.pt
}

Received August 8, 2007; revised January 9, 2008; published online February 15, 2008

\begin{abstract}
We consider matrix Wiener-Hopf plus Hankel operators acting between Lebesgue spaces on the real line with Fourier symbols presenting some even properties (which in particular include unitary matrix-valued functions), and also with Fourier symbols which contain sectorial matrices. In both situations, different conditions are founded to ensure the operators invertibility, one-sided invertibility, Fredholm property, and the so-called $n$ and $d$-normal properties. An example is provided to illustrate the proposed theory.

Key words: matrix Wiener-Hopf plus Hankel operator, invertibility, Fredholm property, unitary matrix-valued function, sectorial matrix-valued function.
\end{abstract}

\section{Introduction and General Framework}

The Wiener-Hopf operators received their name due to the pioneering work of Norbert Wiener and Eberhard Hopf [21] about the study of integral equations of the form

$$
c \varphi(x)+\int_{0}^{+\infty} k(x-y) \varphi(y) d y=f(x), \quad x \in \mathbb{R}_{+},
$$

for an unknown $\varphi$ from $L^{2}\left(\mathbb{R}_{+}\right)$where $f \in L^{2}\left(\mathbb{R}_{+}\right)$is arbitrarily given, and $c \in \mathbb{C}$ and $k \in L^{1}(\mathbb{R})$ are fixed and known. Indeed, from these Wiener-Hopf equations arise the (classical) Wiener-Hopf operators defined by

$$
W_{\phi} f(x)=c f(x)+\int_{0}^{+\infty} k(x-y) f(y) d y, \quad x \in \mathbb{R}_{+},
$$

where $\phi$ belongs to the Wiener algebra

$$
\mathbb{W}=\left\{\phi: \phi=c+\mathcal{F} k, \quad c \in \mathbb{C}, k \in L^{1}(\mathbb{R})\right\}
$$


which is a Banach algebra when endowed with the norm

$$
\|c+\mathcal{F} k\|_{\mathbb{W}}=|c|+\|k\|_{L^{1}(\mathbb{R})}
$$

and the usual multiplication operation. Having in mind the convolution operation, the definition of $W_{\phi}$ in (1.1) gives rise to an understanding of the Wiener-Hopf operators as convolution type operators. Therefore, they can also be represented as

$$
W_{\phi}=r_{+} \mathcal{F}^{-1} \phi \cdot \mathcal{F}: L_{+}^{2}(\mathbb{R}) \rightarrow L^{2}\left(\mathbb{R}_{+}\right),
$$

where $L_{+}^{2}(\mathbb{R})$ denotes the subspace of $L^{2}(\mathbb{R})$ formed by all the functions supported in the closure of $\mathbb{R}_{+}=(0,+\infty), r_{+}$is the restriction operator from $L^{2}(\mathbb{R})$ into $L^{2}\left(\mathbb{R}_{+}\right)$, and $\mathcal{F}$ denotes the Fourier transformation.

Looking now to the structure of the operators in (1.2), we recognize that possibilities other than only the Wiener algebra can be considered for the so-called Fourier symbols $\phi$ of Wiener-Hopf operators. Namely, we may consider to choose $\phi$ among the $L^{\infty}(\mathbb{R})$ elements (i.e., as a measurable essentially bounded function in the real line).

Within the context of (1.1), the Hankel integral operators $H$ have the form

$$
H f(x)=\int_{0}^{+\infty} k(x+y) f(y) d y, \quad x \in \mathbb{R}_{+}
$$

for some $k \in L^{1}(\mathbb{R})$. It is well known that $H$, as an operator defined between $L^{2}$ spaces, is a compact operator. However, as indicated above, it is also possible to provide a rigorous meaning to the expression (1.3) when the kernel $k$ is a temperate distribution whose Fourier transform belongs to $L^{\infty}(\mathbb{R})$.

Although during a long period of time the operators of type (1.1) and type (1.3) were studied separately, in the last years integral equations governed by algebraic sums of Wiener-Hopf and Hankel operators have been receiving increasing attention (cf. $[1,2,4,6,10,12,13,14,15,16,17,18,19,20]$ ). A great part of the interest is directly originated by concrete mathematicalphysics applications where Wiener-Hopf plus Hankel operators appear. This is the case of problems in wave diffraction phenomena which are modeled by boundary-transmission value problems that can be equivalently translated into systems of integral equations characterized by such kind of operators (see, e.g., $[5,7,8])$.

It is therefore easy to understand that a fundamental goal in those studies consists in obtaining conditions that lead to the regularity properties of the Wiener-Hopf plus Hankel operators, i.e., their invertibility, Fredholm property, and other properties that are directly dependent on the kernel and image of the operators.

To be more concrete, let us recall the definitions of some of these concepts. For this purpose, consider a bounded linear operator acting between Banach spaces $T: X \rightarrow Y$. Assuming that $\operatorname{Im} T$ is closed (i.e. $T$ is normally solvable), the cokernel of $T$ can be defined by the quotient Coker $T=Y / \operatorname{Im} T$. Then, $T$ is said to be properly $d$-normal if $\operatorname{dim} \operatorname{Coker} T$ is finite and $\operatorname{dim} \operatorname{Ker} T$ is 
infinite, properly $n$-normal if $\operatorname{dim} \operatorname{Ker} T$ is finite and $\operatorname{dim}$ Coker $T$ is infinite, and Fredholm if both $\operatorname{dim} \operatorname{Ker} T$ and $\operatorname{dim} \operatorname{Coker} T$ are finite. Additionally, we say that $T$ is left-invertible or right-invertible if there exist $T_{l}^{-}: Y \rightarrow X$ or $T_{r}^{-}: Y \rightarrow X$ such that $T_{l}^{-} T=I_{X}$ or $T T_{r}^{-}=I_{Y}$, respectively. As usual, in the case when both $T_{l}^{-}$and $T_{r}^{-}$exist, the operator $T$ is said to be invertible (or both-sided invertible). Alternatively, it can be shown that $T$ is left-invertible if and only if $T$ is injective, normally solvable, and $\operatorname{Im} T$ is a complementable subspace of $Y$. In the same way, $T$ is right-invertible if and only if $T$ is surjective and the (always closed) $\operatorname{Ker} T$ is complementable in $X$.

For some classes of Fourier symbols of Wiener-Hopf plus Hankel operators the regularity properties of the operators are already known. This is the case of the continuous or, even, piecewise continuous functions on the compactificated real line (cf. the above references). Despite those advances, for some other classes of Fourier symbols a complete description of the regularity properties is still missing.

In the present paper, we will work not only with the scalar version of the above operators but with their matrix analogue. Namely, for $N \in \mathbb{N}$, we will consider matrix Wiener-Hopf plus Hankel operators of the form

$$
W H_{\Phi}=W_{\Phi}+H_{\Phi}:\left[L_{+}^{2}(\mathbb{R})\right]^{N} \rightarrow\left[L^{2}\left(\mathbb{R}_{+}\right)\right]^{N},
$$

with $W_{\Phi}=r_{+} \mathcal{F}^{-1} \Phi \cdot \mathcal{F}$ and $H_{\Phi}=r_{+} \mathcal{F}^{-1} \Phi \cdot \mathcal{F} J$, where $J$ is the reflection operator given by the rule $J \Psi(x)=\widetilde{\Psi}(x)=\Psi(-x), x \in \mathbb{R}$, and $\Phi \in\left[L^{\infty}(\mathbb{R})\right]^{N \times N}$. Our goal is to obtain characterizations for the regularity properties of (1.4), in the cases where:

(i) the Fourier symbol presents some even symmetry when combined with its conjugate transpose;

(ii) the Fourier symbol is a matrix function which allows certain factorizations depending on sectorial elements.

Therefore, we will generalize the results of [1], and will also consider other classes of Fourier symbols which were not treated in [1]. We recall that $\Phi \in$ $\left[L^{\infty}(\mathbb{R})\right]^{N \times N}$ is called unitary if $\Phi \Phi^{*}=\Phi^{*} \Phi=I_{N \times N}$, where $\Phi^{*}$ stands for the conjugate transpose of $\Phi$.

\section{Matrix Wiener-Hopf plus Hankel Operators with Sym- metry}

For a Banach algebra $B$, we are going to denote by $\mathcal{G} B$ the group of all invertible elements in $B$. In addition, for

$$
\mathbb{C}_{-}=\{z \in \mathbb{C}: \Im m z<0\}, \quad \mathbb{C}_{+}=\{z \in \mathbb{C}: \Im m z>0\},
$$

we will denote by $H^{\infty}\left(\mathbb{C}_{ \pm}\right)$the set of all bounded and analytic functions in $\mathbb{C}_{ \pm}$. Fatou's theorem ensures that functions in $H^{\infty}\left(\mathbb{C}_{ \pm}\right)$have non-tangential limits on $\mathbb{R}$ almost everywhere. Thus, let $H_{ \pm}^{\infty}:=H_{ \pm}^{\infty}(\mathbb{R})$ be the set of all functions in $L^{\infty}(\mathbb{R})$ that are non-tangential limits of elements in $H^{\infty}\left(\mathbb{C}_{ \pm}\right)$. Moreover, it is well known that $H_{ \pm}^{\infty}$ are closed subalgebras of $L^{\infty}(\mathbb{R})$. Finally, $C(\dot{\mathbb{R}})$ will 
denote the space of all continuous (complex-valued) functions on $\mathbb{R}$ for which both limits at $\pm \infty$ exist and coincide.

In the Wiener-Hopf operators case there is a well known theorem - due to Ronald G. Douglas and Donald Sarason - about the regularity properties of this kind of operators and the distances from the Fourier symbols to certain spaces. More precisely, the theorem may be written in the following form.

Theorem 1 [Douglas and Sarason [11]]. If $\Phi \in\left[L^{\infty}(\mathbb{R})\right]^{N \times N}$ is unitary, then:

(a) $W_{\Phi}$ is invertible if and only if $\operatorname{dist}\left(\Phi, \mathcal{G}\left[H_{+}^{\infty}\right]^{N \times N}\right)<1$ or if and only if $\operatorname{dist}\left(\Phi, \mathcal{G}\left[H_{-}^{\infty}\right]^{N \times N}\right)<1$

(b) $W_{\Phi}$ is left-invertible if and only if $\operatorname{dist}\left(\Phi,\left[H_{+}^{\infty}\right]^{N \times N}\right)<1$;

$\left(b^{\prime}\right) W_{\Phi}$ is right-invertible if and only if $\operatorname{dist}\left(\Phi,\left[H_{-}^{\infty}\right]^{N \times N}\right)<1$;

(c) $W_{\Phi}$ is Fredholm if and only if $\operatorname{dist}\left(\Phi, \mathcal{G}\left[C(\dot{\mathbb{R}})+H_{+}^{\infty}\right]^{N \times N}\right)<1$ or if and only if $\operatorname{dist}\left(\Phi, \mathcal{G}\left[C(\dot{\mathbb{R}})+H_{-}^{\infty}\right]^{N \times N}\right)<1$;

(d) $W_{\Phi}$ is $n$-normal if and only if $\operatorname{dist}\left(\Phi,\left[C(\dot{\mathbb{R}})+H_{+}^{\infty}\right]^{N \times N}\right)<1$;

(d') $W_{\Phi}$ is d-normal if and only if $\operatorname{dist}\left(\Phi,\left[C(\dot{\mathbb{R}})+H_{-}^{\infty}\right]^{N \times N}\right)<1$.

The last theorem served as a motivation for obtaining such kind of result for our Wiener-Hopf plus Hankel operators. However, it is clear that adding Hankel operators to the above Wiener-Hopf operators will influence several changes in the regularity properties of the resulting operators. In addition, we will work not only with unitary matrix-valued functions but with the more general class which appears in the general assumption of the next result.

Theorem 2. Let $\Phi \in \mathcal{G}\left[L^{\infty}(\mathbb{R})\right]^{N \times N}$ and assume that $\Phi^{*} \Phi$ is an even matrixvalued function.

(a) $W H_{\Phi}$ and $W_{\Phi}-H_{\Phi}$ are invertible if and only if

$$
\operatorname{dist}\left(\widetilde{\Phi \Phi^{-1}}, \mathcal{G}\left[H_{+}^{\infty}\right]^{N \times N}\right)<1
$$

or if and only if $\operatorname{dist}\left(\widetilde{\Phi \Phi^{-1}}, \mathcal{G}\left[H_{-}^{\infty}\right]^{N \times N}\right)<1$.

(b) $W H_{\Phi}$ and $W_{\Phi}-H_{\Phi}$ are left-invertible if and only if

$$
\operatorname{dist}\left(\Phi \widetilde{\Phi-1},\left[H_{+}^{\infty}\right]^{N \times N}\right)<1 .
$$

(b') $W H_{\Phi}$ and $W_{\Phi}-H_{\Phi}$ are right-invertible if and only if

$$
\operatorname{dist}\left(\Phi \widetilde{\Phi^{-1}},\left[H_{-}^{\infty}\right]^{N \times N}\right)<1 .
$$


(c) $W H_{\Phi}$ and $W_{\Phi}-H_{\Phi}$ are Fredholm if and only if

$$
\operatorname{dist}\left(\widetilde{\Phi \Phi^{-1}}, \mathcal{G}\left[C(\dot{\mathbb{R}})+H_{+}^{\infty}\right]^{N \times N}\right)<1
$$

or if and only if $\operatorname{dist}\left(\widetilde{\Phi \Phi^{-1}}, \mathcal{G}\left[C(\dot{\mathbb{R}})+H_{-}^{\infty}\right]^{N \times N}\right)<1$.

(d) $W H_{\Phi}$ and $W_{\Phi}-H_{\Phi}$ are n-normal if and only if

$$
\operatorname{dist}\left(\Phi \widetilde{\Phi^{-1}},\left[C(\dot{\mathbb{R}})+H_{+}^{\infty}\right]^{N \times N}\right)<1 .
$$

(d') $W H_{\Phi}$ and $W_{\Phi}-H_{\Phi}$ are d-normal if and only if

$$
\operatorname{dist}\left(\Phi \widetilde{\Phi^{-1}},\left[C(\dot{\mathbb{R}})+H_{-}^{\infty}\right]^{N \times N}\right)<1 .
$$

Proof The proof is based on the notion of $\Delta$-relation after extension (introduced in [3]), and which we will now recall. For bounded linear operators acting between Banach spaces, e.g. $T: X_{1} \rightarrow X_{2}$ and $S: Y_{1} \rightarrow Y_{2}$, we say that $T$ is $\Delta$-related after extension to $S$ if there is an auxiliary bounded linear operator acting between Banach spaces $T_{\Delta}: X_{1 \Delta} \rightarrow X_{2 \Delta}$, and bounded invertible operators $E$ and $F$ such that

$$
\left[\begin{array}{cc}
T & 0 \\
0 & T_{\Delta}
\end{array}\right]=E\left[\begin{array}{cc}
S & 0 \\
0 & I_{Z}
\end{array}\right] F
$$

where $Z$ is an additional Banach space and $I_{Z}$ represents the identity operator in $Z$. In the particular case where

$$
T_{\Delta}=I_{X_{1 \Delta}}: X_{1 \Delta} \rightarrow X_{2 \Delta}=X_{1 \Delta}
$$

is the identity operator, we say that $T$ and $S$ are equivalent after extension operators.

From [3] we can derive that $T=W H_{\Phi}$ is $\Delta$-related after extension to the Wiener-Hopf operator $S=W_{\Phi \widetilde{\Phi^{-1}}}$, and with $T_{\Delta}=W_{\Phi}-H_{\Phi}$ in (2.1). Thus, we are now going to analyze the Fourier symbol $\Phi \widetilde{\Phi^{-1}}$.

Let us observe that for $\Phi \in \mathcal{G}\left[L^{\infty}(\mathbb{R})\right]^{N \times N}$ the function $\Phi \widetilde{\Phi^{-1}}$ is unitary valued if and only if $\Phi^{*} \Phi$ is even. Indeed, suppose that $\Phi^{*} \Phi$ is even. By the definition we have:

$$
\Phi^{*} \Phi=\widetilde{\Phi^{*}} \widetilde{\Phi}
$$

From here it directly follows that

$$
\Phi \widetilde{\Phi^{-1}}=\left(\Phi^{*}\right)^{-1} \widetilde{\Phi^{*}} .
$$

To simplify further arguments, let us introduce the new notation: $\Psi:=\Phi \widetilde{\Phi^{-1}}$. To prove that $\Psi$ is unitary valued we have to show that

$$
\Psi \Psi^{*}=\Psi^{*} \Psi=I_{N \times N} .
$$

Math. Model. Anal., 13(1):7-16, 2008. 
Performing a direct substitution, we will have that

$$
\Psi \Psi^{*}=\Phi \widetilde{\Phi-1}\left(\widetilde{\Phi^{-1}}\right)^{*} \Phi^{*}
$$

Having in mind formula (2.2), from the last equality one obtains:

$\Psi \Psi^{*}=\left(\Phi^{*}\right)^{-1}\left(\widetilde{\Phi^{*}}\right)\left(\widetilde{\Phi^{-1}}\right)^{*} \Phi^{*}=\left(\Phi^{*}\right)^{-1}\left(\widetilde{\Phi^{*}}\right)\left(\widetilde{\Phi^{*}}\right)^{-1} \Phi^{*}=\left(\Phi^{*}\right)^{-1} \Phi^{*}=I_{N \times N}$.

Analogously, we have:

$$
\Psi^{*} \Psi=\left(\widetilde{\Phi^{-1}}\right)^{*} \Phi^{*} \Phi \widetilde{\Phi^{-1}}=\left(\widetilde{\Phi^{-1}}\right)^{*} \Phi^{*}\left(\Phi^{*}\right)^{-1} \widetilde{\Phi^{*}}=I_{N \times N} .
$$

To prove the above stated equivalence we are left to show that if $\Phi \widetilde{\Phi^{-1}}$ is a unitary matrix-valued function, then $\Phi^{*} \Phi$ is even. If $\Phi \widehat{\Phi^{-1}}$ is unitary, then we derive that

$$
\Phi \widetilde{\Phi^{-1}}\left(\widetilde{\Phi^{-1}}\right)^{*} \Phi^{*}=I_{N \times N}
$$

Consequently, we have:

$$
\Phi \widetilde{\Phi^{-1}}=\left(\Phi^{*}\right)^{-1} \widetilde{\Phi^{*}}
$$

Hence, $\Phi^{*} \Phi=\widetilde{\Phi^{*}} \widetilde{\Phi}$ and we have shown the above announced equivalence.

From the above reasoning we have that $\Phi \widetilde{\Phi^{-1}}$ is unitary. We can now apply Theorem 1 to the operator $W_{\Phi \widetilde{\Phi-1}}$ and obtain all the above stated conditions in terms of distances. Now, the result follows if we interpret (2.1) as an equivalence after extension relation between $\operatorname{diag}\left[T, T_{\Delta}\right]=\operatorname{diag}\left[W H_{\Phi}, W_{\Phi}-\right.$ $\left.H_{\Phi}\right]$ and $S=W_{\Phi \widetilde{\Phi^{-1}}}$, which allows the transfer of regularity properties from $W_{\Phi \Phi-1}$ to $W H_{\Phi}$ and $W_{\Phi}-H_{\Phi}$.

Remark 1. Note that the global assumption of the last theorem which requires that $\Phi^{*} \Phi$ is an even matrix-valued function is more general than assuming $\Phi$ to be an unitary matrix-valued function.

\section{Matrix Wiener-Hopf plus Hankel Operators with Sec- torial Components}

In the present section we will work out a different characterization for the regularity properties of matrix Wiener-Hopf plus Hankel operators, and which is now based on the use of certain sectorial parts of the matrix Fourier symbols of the operators.

Definition 1. A matrix function $S \in\left[L^{\infty}(\mathbb{R})\right]^{N \times N}$ is said to be sectorial if there exist a real number $\varepsilon>0$ and two (constant) matrices $B, C \in \mathcal{G} \mathbb{C}^{N \times N}$ such that

$$
\Re e(B S(x) C z, z) \geq \varepsilon\|z\|^{2},
$$

for almost all $x \in \mathbb{R}$ and all $z \in \mathbb{C}^{N}$. 
We will denote by $\mathcal{S}^{N \times N}$ the set of all sectorial matrix functions (in $\left.\left[L^{\infty}(\mathbb{R})\right]^{N \times N}\right)$. Once again, for matrix Wiener-Hopf operators with such kind of Fourier symbols a description of the possible regularity properties is known.

Theorem 3. [9] If $\Phi \in \mathcal{G}\left[L^{\infty}(\mathbb{R})\right]^{N \times N}$, then:

(a) $W_{\Phi}$ is invertible if and only if $\Phi=S h, S \in \mathcal{S}^{N \times N}, h \in \mathcal{G}\left[H_{+}^{\infty}\right]^{N \times N}$ or if and only if $\Phi=h S, S \in \mathcal{S}^{N \times N}, h \in \mathcal{G}\left[H_{-}^{\infty}\right]^{N \times N}$;

(b) $W_{\Phi}$ is left-invertible if and only if $\Phi=S h, S \in \mathcal{S}^{N \times N}, h \in\left[H_{+}^{\infty}\right]^{N \times N}$;

(b') $W_{\Phi}$ is right-invertible if and only if $\Phi=h S, S \in \mathcal{S}^{N \times N}, h \in\left[H_{-}^{\infty}\right]^{N \times N}$;

(c) $W_{\Phi}$ is Fredholm if and only if $\Phi=S h, S \in \mathcal{S}^{N \times N}, h \in \mathcal{G}[C(\dot{\mathbb{R}})+$ $\left.H_{+}^{\infty}\right]^{N \times N}$ or if and only if $\Phi=h S, S \in \mathcal{S}^{N \times N}, h \in \mathcal{G}\left[C(\dot{\mathbb{R}})+H_{-}^{\infty}\right]^{N \times N}$;

(d) $W_{\Phi}$ is n-normal if and only if $\Phi=S h, S \in \mathcal{S}^{N \times N}, h \in[C(\dot{\mathbb{R}})+$ $\left.H_{+}^{\infty}\right]^{N \times N}$

(d') $W_{\Phi}$ is d-normal if and only if $\Phi=h S, S \in \mathcal{S}^{N \times N}, h \in[C(\dot{\mathbb{R}})+$ $\left.H_{-}^{\infty}\right]^{N \times N}$.

We will now introduce a corresponding theorem for Wiener-Hopf plus Hankel operators (with the help of Wiener-Hopf minus Hankel operators $W_{\Phi}-H_{\Phi}$ ).

Theorem 4. Let $\Phi \in \mathcal{G}\left[L^{\infty}(\mathbb{R})\right]^{N \times N}$.

(a) $W H_{\Phi}$ and $W_{\Phi}-H_{\Phi}$ are both invertible if and only if

$$
\Phi \widetilde{\Phi-1}=S h, S \in \mathcal{S}^{N \times N}, h \in \mathcal{G}\left[H_{+}^{\infty}\right]^{N \times N}
$$

or if and only if $\Phi \widetilde{\Phi \Phi^{-1}}=h S, S \in \mathcal{S}^{N \times N}, h \in \mathcal{G}\left[H_{-}^{\infty}\right]^{N \times N}$.

(b) $W H_{\Phi}$ and $W_{\Phi}-H_{\Phi}$ are both left-invertible if and only if

$$
\widetilde{\Phi \Phi^{-1}}=S h, S \in \mathcal{S}^{N \times N}, h \in\left[H_{+}^{\infty}\right]^{N \times N} .
$$

(c) $W H_{\Phi}$ and $W_{\Phi}-H_{\Phi}$ are both right-invertible if and only if

$$
\widetilde{\Phi \Phi^{-1}}=h S, S \in \mathcal{S}^{N \times N}, h \in\left[H_{-}^{\infty}\right]^{N \times N} .
$$

(d) $W H_{\Phi}$ and $W_{\Phi}-H_{\Phi}$ are both Fredholm if and only if

$$
\widetilde{\Phi \Phi^{-1}}=S h, S \in \mathcal{S}^{N \times N}, h \in \mathcal{G}\left[C(\dot{\mathbb{R}})+H_{+}^{\infty}\right]^{N \times N}
$$

or if and only if

$$
\Phi \widetilde{\Phi-1}=h S, S \in \mathcal{S}^{N \times N}, h \in \mathcal{G}\left[C(\dot{\mathbb{R}})+H_{-}^{\infty}\right]^{N \times N} .
$$


(e) $W H_{\Phi}$ and $W_{\Phi}-H_{\Phi}$ are both n-normal if and only if

$$
\widetilde{\Phi \Phi^{-1}}=S h, S \in \mathcal{S}^{N \times N}, h \in\left[C(\dot{\mathbb{R}})+H_{+}^{\infty}\right]^{N \times N} .
$$

(f) $W H_{\Phi}$ and $W_{\Phi}-H_{\Phi}$ are both d-normal if and only if

$$
\Phi \widetilde{\Phi^{-1}}=h S, S \in \mathcal{S}^{N \times N}, h \in\left[C(\dot{\mathbb{R}})+H_{-}^{\infty}\right]^{N \times N} .
$$

Proof To prove this result we perform the same reasoning as in the proof of Theorem 2, and make use of the fact that in the $\Delta$-relation after extension we have $T_{\Delta}=W_{\Phi}-H_{\Phi}$. This allows us to transfer the above mentioned regularity properties from the operator $W_{\Phi \widetilde{\Phi-1}}$ to operator $\operatorname{diag}\left[W H_{\Phi}, W_{\Phi}-H_{\Phi}\right]$ by using Theorem 3 and the indicated operator relation.

We assemble in the next corollary the part of Theorem 4 which is the most practical conclusion of the present work.

Corollary 1. Let $\Phi \in \mathcal{G}\left[L^{\infty}(\mathbb{R})\right]^{N \times N}$.

(a) If $\Phi \widetilde{\Phi \Phi^{-1}}=S h, s \in \mathcal{S}^{N \times N}$ and $h \in \mathcal{G}\left[H_{+}^{\infty}\right]^{N \times N}$, then $W H_{\Phi}$ is invertible.

$\left(\mathrm{a}^{\prime}\right)$ If $\Phi \widetilde{\Phi^{-1}}=h S, s \in \mathcal{S}^{N \times N}$ and $h \in \mathcal{G}\left[H_{-}^{\infty}\right]^{N \times N}$, then $W H_{\Phi}$ is invertible.

(b) If $\Phi \widetilde{\Phi-1}=S h$, with $S \in \mathcal{S}^{N \times N}$ and $h \in\left[H_{+}^{\infty}\right]^{N \times N}$, then $W H_{\Phi}$ is left-invertible.

(c) If $\Phi \widetilde{\Phi^{-1}}=h S$, with $S \in \mathcal{S}^{N \times N}$ and $h \in\left[H_{-}^{\infty}\right]^{N \times N}$, then $W H_{\Phi}$ is rightinvertible.

(d) If $\Phi \widetilde{\Phi-1}=S h$, with $S \in \mathcal{S}^{N \times N}$ and $h \in \mathcal{G}\left[C(\dot{\mathbb{R}})+H_{+}^{\infty}\right]^{N \times N}$, then $W H_{\Phi}$ is Fredholm.

$\left(\mathrm{d}^{\prime}\right)$ If $\Phi \widetilde{\Phi^{-1}}=h S$, with $S \in \mathcal{S}^{N \times N}$ and $h \in \mathcal{G}\left[C(\dot{\mathbb{R}})+H_{-}^{\infty}\right]^{N \times N}$, then $W H_{\Phi}$ is Fredholm.

(e) If $\Phi \widetilde{\Phi-1}=S h$, with $S \in \mathcal{S}^{N \times N}, h \in\left[C(\dot{\mathbb{R}})+H_{+}^{\infty}\right]^{N \times N}$, then $W H_{\Phi}$ is n-normal.

(f) If $\Phi \widetilde{\Phi^{-1}}=h S$, with $S \in \mathcal{S}^{N \times N}, h \in\left[C(\dot{\mathbb{R}})+H_{-}^{\infty}\right]^{N \times N}$, then $W H_{\Phi}$ is d-normal.

In this final part we would like to present an example which shows the applicability of the last result. Let us consider the matrix Wiener-Hopf plus Hankel operator

$$
W H_{\Phi_{p}}:\left[L_{+}^{2}(\mathbb{R})\right]^{2} \rightarrow\left[L^{2}\left(\mathbb{R}_{+}\right)\right]^{2},
$$

with the particular Fourier symbol

$$
\Phi_{p}(x)=\left(\begin{array}{cc}
2+\sin x & 0 \\
\cos x & 1
\end{array}\right)\left(\begin{array}{cc}
e^{i \alpha x} & 0 \\
0 & 1
\end{array}\right), \quad x \in \mathbb{R},
$$


where $\alpha \in \mathbb{R}$ is a given parameter. Direct computations show that

$$
\Phi_{p}(x) \widetilde{\Phi_{p}^{-1}}(x)=\left(\begin{array}{cc}
e^{2 i \alpha x} & 0 \\
0 & 1
\end{array}\right)\left(\begin{array}{cc}
(2+\sin x)(2-\sin x)^{-1} & 0 \\
\left(e^{2 i \alpha x}-1\right) \cos x(2-\sin x)^{-1} & 1
\end{array}\right)=: h_{p} S_{p},
$$

and

$$
\Phi_{p}(x) \widetilde{\Phi_{p}^{-1}}(x)=\left(\begin{array}{cc}
(2+\sin x)(2-\sin x)^{-1} & 0 \\
\left(1-e^{-2 i \alpha x}\right) \cos x(2-\sin x)^{-1} & 1
\end{array}\right)\left(\begin{array}{cc}
e^{2 i \alpha x} & 0 \\
0 & 1
\end{array}\right)=: s_{p} H_{p} .
$$

So, we have that $s_{p}$ and $S_{p}$ are sectorial matrix functions because the main minors of that matrices are positive definite and this is a particular case of sectorial matrix functions. Moreover, depending whether $\alpha \geq 0$ or $\alpha \leq 0$, we have $h_{p}, H_{p} \in H_{+}^{\infty}$ or $h_{p}, H_{p} \in H_{-}^{\infty}$, respectively. Therefore, applying Corollary 1 , we conclude that:

(a) if $\alpha=0$, then $W H_{\Phi_{p}}$ is invertible;

(b) if $\alpha>0$, then $W H_{\Phi_{p}}$ is left-invertible;

(c) if $\alpha<0$, then $W H_{\Phi_{p}}$ is right-invertible.

\section{Acknowledgement}

This work was supported in part by Unidade de Investigação Matemática e Aplicações of Universidade de Aveiro through the Portuguese Science Foundation (FCT-Fundação para a Ciência e a Tecnologia).

\section{References}

[1] G. Bogveradze and L.P. Castro. Wiener-Hopf plus Hankel operators on the real line with unitary and sectorial symbols. Contemp. Math., 414:77-85, 2006.

[2] G. Bogveradze and L.P. Castro. On the Fredholm index of matrix Wiener-Hopf plus/minus Hankel operators with semi-almost periodic symbols. Oper. Theory Adv. Appl., 181, 2008. (16 pp., to appear)

[3] L.P. Castro and F.-O. Speck. Regularity properties and generalized inverses of delta-related operators. Z. Anal. Anwendungen, 17:577-598, 1998.

[4] L.P. Castro and F.-O. Speck. Inversion of matrix convolution type operators with symmetry. Port. Math. (N.S.), 62:193-216, 2005.

[5] L.P. Castro, F.-O. Speck and F.S. Teixeira. Explicit solution of a DirichletNeumann wedge diffraction problem with a strip. J. Integral Equations Appl., 15:359-383, 2003.

[6] L.P. Castro, F.-O. Speck and F.S. Teixeira. A direct approach to convolution type operators with symmetry. Math. Nachr., 269/270:73-85, 2004.

[7] L.P. Castro, F.-O. Speck and F.S. Teixeira. On a class of wedge diffraction problems posted by Erhard Meister. Oper. Theory Adv. Appl., 147:213-240, 2004 .

Math. Model. Anal., 13(1):7-16, 2008. 
[8] L.P. Castro, F.-O. Speck and F.S. Teixeira. Mixed boundary value problems for the Helmholtz equation in a quadrant. Integral Equations Operator Theory, 56:1-44, 2006.

[9] K. Clancey and I. Gohberg. Factorization of Matrix Functions and Singular Integral Operators. Birkhäuser Verlag, Basel, 1981.

[10] A.C. Conceição, V.G. Kravchenko and F.S. Teixeira. Factorization of some classes of matrix functions and the resolvent of a Hankel operator. In Factorization, Singular Operators and Related Problems, pp. 101-110. Kluwer Acad. Publ., Dordrecht, 2003.

[11] R.G. Douglas and D. Sarason. Fredholm Toeplitz operators. In Proc. Amer. Math. Soc., volume 26, pp. 117-120, 1970.

[12] T. Ehrhardt. Invertibility theory for Toeplitz plus Hankel operators and singular integral operators with flip. J. Funct. Anal., 208:64-106, 2004.

[13] N. Karapetiants and S. Samko. Equations with Involutive Operators. Birkhäuser, Boston, 2001.

[14] V.G. Kravchenko, A.B. Lebre, G.S. Litvinchuk and F.S. Teixeira. Fredholm theory for a class of singular integral operators with Carleman shift and unbounded coefficients. Math. Nachr., 172:199-210, 1995.

[15] V.G. Kravchenko and G.S. Litvinchuk. Introduction to the Theory of Singular Integral Operators with Shift. Kluwer Academic Publishers Group, Dordrecht, 1994.

[16] A.B. Lebre, E. Meister and F.S. Teixeira. Some results on the invertibility of Wiener-Hopf-Hankel operators. Z. Anal. Anwendungen, 11:57-76, 1992.

[17] A.P. Nolasco and L.P. Castro. A criterion for lateral invertibility of matrix Wiener-Hopf plus Hankel operators with good Hausdorff sets. (9 pp., to appear)

[18] A.P. Nolasco and L.P. Castro. Factorization theory for Wiener-Hopf plus Hankel operators with almost periodic symbols. Contemp. Math., 414:111-128, 2006.

[19] A.P. Nolasco and L.P. Castro. ADuduchava-Saginashvili's type theory for Wiener-Hopf plus Hankel operators. J. Math. Anal. Appl., 331:329-341, 2007.

[20] F.S. Teixeira. On a class of Hankel operators: Fredholm properties and invertibility. Integral Equations Operator Theory, 12:592-613, 1989.

[21] N. Wiener and E. Hopf. Über eine Klasse Singulärer Integralgleichungen. Sitzungsber. Preuß. Akad. Wiss., Phys.-Math., 30/32:696-706, 1931. 